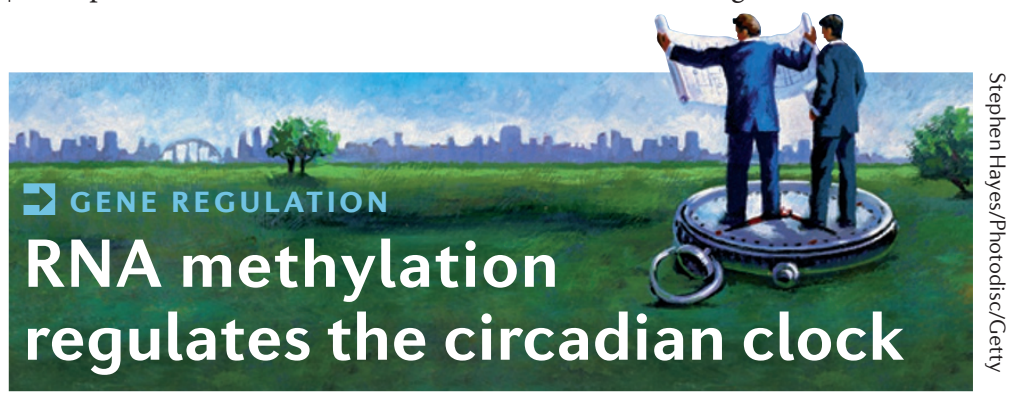

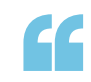

\section{one of the}

first studies to determine a functional consequence of RNA methylation
New methods have recently identified $N^{6}$-methyladenosine $\left(\mathrm{m}^{6} \mathrm{~A}\right)$ RNA transmethylation as the most prevalent internal modification of mRNA, although its function has remained obscure. A new study now reveals that $\mathrm{m}^{6} \mathrm{~A}$ RNA transmethylation is involved in circadian clock regulation.

Recent studies have shown that only one-fifth of 'rhythmic genes' that are involved in the circadian clock are driven by de novo transcription, which suggests that RNA processing and modifications such as transmethylation are relevant to clock function. RNA transmethylation is sensitive to the amount of S-adenosyl-L-methionine (SAM) and S-adenosyl-L-homocysteine (SAH): SAM is a methyl donor co-substrate, whereas $\mathrm{SAH}$ is the by-product of transmethylation that also acts as a competitive inhibitor of the reaction. The ratio of SAM to SAH constitutes the methylation potential of a cell, and inhibition of SAH hydrolysis leads to a build-up of SAH and thus to a decrease in methylation potential.

Fustin et al. used 3-deazaadenosine (DAA), which is an inhibitor of SAH hydrolysis, in cell culture and in mice to determine the effect of decreasing RNA methylation on the circadian clock. They found that DAA caused an elongation of the circadian period.

The authors then used RNA sequencing to ascertain the effect of DAA-induced decrease in methylation potential on the RNA transcriptome. One of the most significant groups of transcripts that were affected, as determined by gene ontology analysis, was those from genes that are involved in rhythmic processes, including several well-known circadian clock genes. RNA-processing genes were also found to be severely dysregulated — in particular, $\mathrm{m}^{6} \mathrm{~A}$ demethylases were upregulated. Subsequent RNAimmunoprecipitation experiments using an anti- $\mathrm{m}^{6} \mathrm{~A}$ antibody followed by RNA sequencing showed that $\mathrm{m}^{6} \mathrm{~A}$ sites were enriched in several clock genes and that $\mathrm{m}^{6} \mathrm{~A}$ methylation was specifically inhibited by DAA.

To investigate the effect of this $\mathrm{m}^{6} \mathrm{~A}$ methylation inhibition on RNA processing dynamics, the authors used pulse-chase RNA-labelling click cytochemistry, which fluorescently labels newly synthesized RNA and allows its subcellular localization to be tracked. DAA was found to cause prolonged nuclear retention of RNA and therefore delay RNA processing, which suggests a mechanism to explain the observed elongated circadian period.

In support of this hypothesis, the authors knocked down METTL3 (methyltransferase-like 3), which encodes the SAM-binding subunit of the only known $\mathrm{m}^{6} \mathrm{~A}$ methyltransferase, and found that it also elongated the circadian period. Moreover, overexpression of METTL3 caused a shortening of the circadian period. Importantly, similarly to DAA inhibition, suppression of METTL3 expression caused slower RNA processing, as shown by pulse-chase click cytochemistry experiments. In particular, knockdown of METTL3 delayed the nuclear exit of the mRNAs of the clock genes ARNTL (aryl hydrocarbon receptor nuclear translocator-like) and PER2 (period circadian clock 2). Thus, suppression of $\mathrm{m}^{6} \mathrm{~A}$ methylation and its effects on RNA processing are sufficient to slow down the circadian clock.

This is one of the first studies to determine a functional consequence of RNA methylation and to uncover its crucial role in circadian clock regulation. Several further questions remain, including the specific roles of RNA splicing, export, stability and translation in this process.

Isabel Lokody 